\title{
ESTRATEGIAS DE SUBSISTENCIA DE CAZADORES RECOLECTORES DE ISLA DAWSON (ESTRECHO DE MAGALLANES) DURANTE LA SEGUNDA MITAD DEL HOLOCENO: PRIMERAS APROXIMACIONES
}

\author{
DOMINIQUE LEGOUPIL", PHILIPPE BÉAREZ** CHRISTINE LEFÈVRE*, \\ MANUEL SAN ROMÁN ${ }^{\cdots *}$ Y Y JIMENA TORRES"
}

\begin{abstract}
RESUMEN
El desarrollo de una prospección arqueológica en la costa de isla Dawson e isla Wickham, ubicadas en una posición estratégica entre las diferentes vías de comunicación que conectan la porción central del estrecho de Magallanes con el archipiélago fueguino, registró 27 yacimientos que corresponden a antiguos campamentos de cazadores-recolectores marinos que habitaron la zona. La realización de excavaciones de sondeo en seis de estos sitios permitió recuperar un interesante conjunto de restos arqueofaunísticos. A partir del análisis de éstos conjuntos, describimos las principales características observadas y ofrecemos un primer panorama referido a la subsistencia de los grupos que habitaron esta zona del archipiélago fueguino.
\end{abstract}

PALABRAS CLAVE: isla Dawson, estrecho de Magallanes, Holoceno tardío, cazadores marinos, pesca, subsistencia.

\section{SUBSISTENCE STRATEGIES OF HUNTER GATHERERS FROM DAWSON ISLAND (MAGELLAN'S STRAIT) DURING THE SECOND HALF OF THE HOLOCENE: FIRST APPROXIMATIONS}

\begin{abstract}
The development of an archaeological survey on the coast of Dawson and Wickham island, located in a strategic position between different path roads that connect the central portion of the Magellan strait with the fueguian archipelago, allowed detecting 27 sites that correspond to former marine hunter-gatherer camps of the inhabitants of the zone. The accomplishment of excavations in six of these sites allowed recovering an interesting set of archaeological fauna remains. From the analysis of these assemblages, we describe the main observed characteristics and offer a first panorama referred to the subsistence of the groups that lived in this zone of the fueguian archipelago.
\end{abstract}

KEY WORDS: Dawson island, Magellan strait, Late Holocene, marine hunters, fishing, subsistence.

• UMR 7041, CNRS (Francia), dominique.legoupil@mae.u-paris10.fr, jimena.torres@malix.univ-paris1.fr.

* UMR 7209, CNRS (Francia), bearez@mnhn.fr, lefevre@mnhn.fr.

*** Centro de Estudios del Hombre Austral, Instituto de la Patagonia, Universidad de Magallanes (Chile). manuel.sanroman@ umag.cl. 


\section{INTRODUCCIÓN}

La isla Dawson, en medio del estrecho de Magallanes, pertenece al ámbito de los indígenas canoeros. La subsistencia de estos cazadores nómades, instalados en los archipiélagos de Patagonia y Tierra de Fuego desde hace más de seis milenios, se basaba en los moluscos, los mamíferos marinos y las aves, como lo indican numerosos relatos de navegantes y etnólogos. Sin embargo su sistema de aprovisionamiento, en parte programado y especializado (a menudo en función de las estaciones) y en parte aleatorio (encuentros al azar) es aún mal conocido (Legoupil 2000).

Una prospección realizada sobre la isla Dawson, fue la ocasión de enriquecer nuestros conocimientos sobre los distintos métodos de adquisición alimentaria de los grupos que frecuentaban esta gran isla desde hace al menos 4000 años. Es por la incorporación de tales datos y su comparación

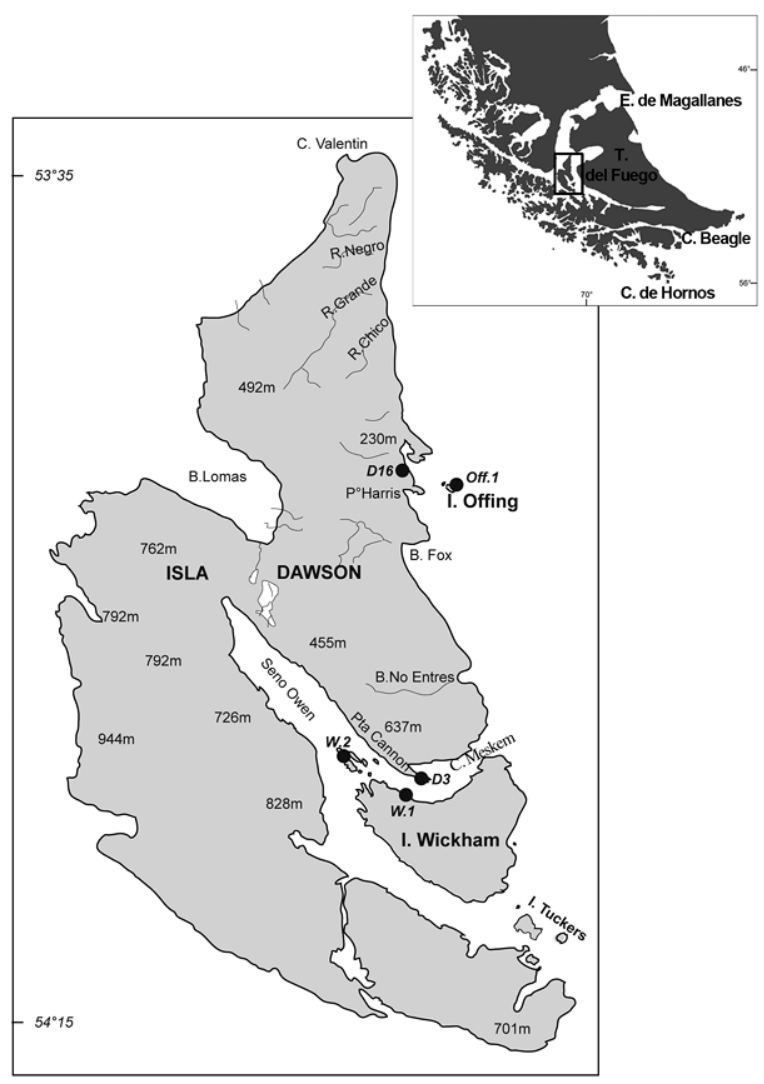

Fig. 1. Mapa de ubicación de los sitios arqueológicos sondeados. con las observaciones obtenidas en otras regiones de los archipiélagos, que podremos un día seguramente reconstituir estos sistemas de subsistencia y los ritmos de movilidad que le estaban vinculados.

La prospección permitió detectar 27 sitios sobre la costa de isla Dawson y las islas satélites Wickham y Offing (Legoupil et al. 2007, Legoupil et al. 2011). Uno de estos yacimientos, denominado Offing 2 , es objeto de excavaciones extensivas desde el año 2008, en el marco de un programa de investigación franco-chileno (Misión arqueológica francesa y FONDECYT 1085329).

En el marco de la prospección se efectuaron excavaciones de sondeo en 5 yacimientos considerados como los sitios más importantes (Fig. 1). Estos se sitúan en dos zonas: la zona central (incluyendo los sitios Dawson 16 y Offing 1) y la zona sur con los sitios Dawson 3 (donde se observó dos ocupaciones en dos terrazas marinas diferentes), y Wickham 1 y 2 (ibid). La mayoría de los yacimientos sondeados (Dawson 16, Dawson 3 sondeo A30, Wickham 1 y 2) se encuentran a poca elevación sobre el nivel del mar $\left(\leq 2 \mathrm{msnm}^{1}\right)$, hecho que a priori permite adscribirlos al Holoceno tardío (cf. San Román et al. 2009).

Otro yacimiento, Offing 1 , no presentaba ningún indicador cronológico, pero su posición sobre una terraza ligeramente más elevada (3,20 msnm), permite sugerir una cronología un poco más antigua. Finalmente, el sitio Dawson 3, emplazado sobre la terraza a $4 \mathrm{msnm}$, proporcionó un fechado de 3090 $\pm 56 \mathrm{AP}$ (Erl-10956) para la base del sondeo A1/A2.

Estos sondeos proporcionaron datos suficientes (más de dos mil restos óseos determinados) para permitir una primera aproximación sobre las estrategias de explotación de los recursos alimentarios de estos grupos.

\section{LOS RECURSOS DISPONIBLES}

La isla Dawson, dada su calidad de zona militar, constituye un refugio protegido para la fauna. Nos ofrece así una visión de lo que fue probablemente la riqueza ecológica de los archipiélagos, destacando la facilidad para acercarse de animales que han sido poco molestados por el hombre. Sobre la costa este, en la Punta Arska y en la isla Offing,

1 Metros sobre el nivel del mar. 


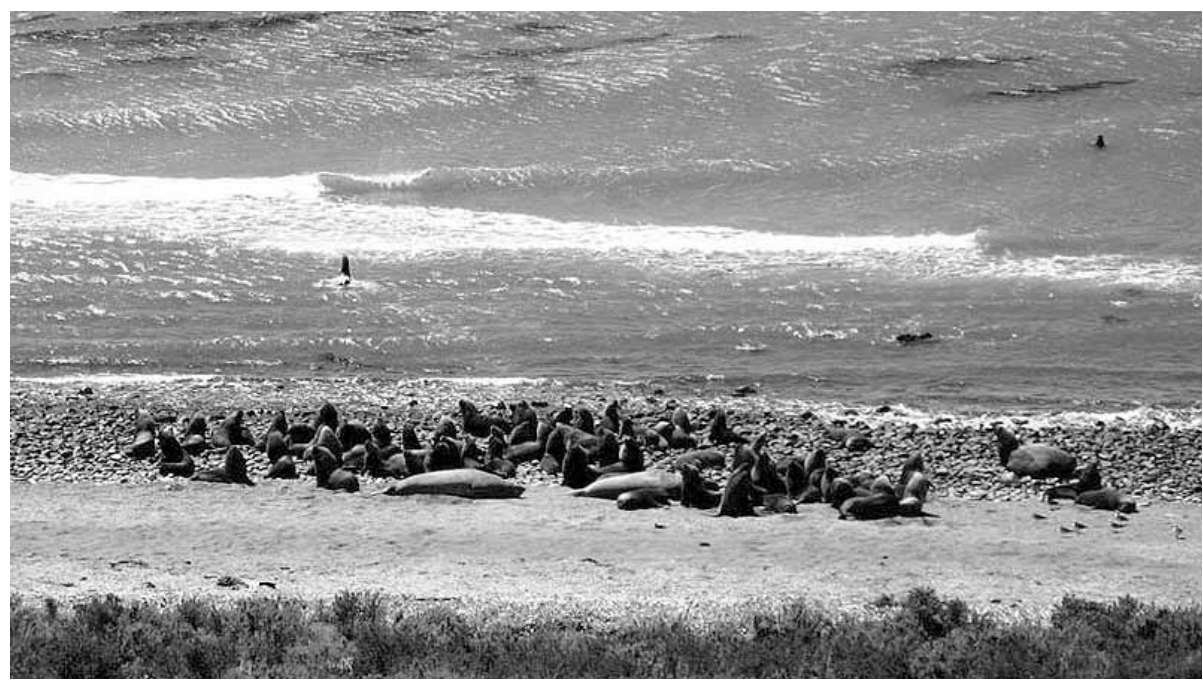

Fig. 2. La lobería de Punta Arska. En el centro se observan dos machos de Mirounga leonina.(D. Legoupil).

se pudo observar dos apostaderos de pinnípedos (Otaria flavescens y Arctocephalus australis) entre los cuales se mezclaban algunos elefantes de mar (Mirounga leonina) (Fig. 2). Los delfines abundan en las bahías próximas a Puerto Harris, y durante la prospección, pudimos avistar orcas a lo largo de la costa de la isla Wickham (Fig. 3). Las aves marinas son innumerables, especialmente sobre los pequeños islotes (Offing, Tesner, Tucker).

Esta riqueza faunística se encuentra expresada en el espectro alimentario muy amplio, típico de los indígenas canoeros, representado en los yacimientos detectados.

\section{LA ACTIVIDAD CINEGÉTICA: LOS DATOS}

La caza en los diferentes sitios sondeados esta evidenciada por un número significativo de restos óseos (Tabla 1). Dentro de los 3025 restos estudiados, 474 eran de mamíferos cuyo 94\% fueron determinados al nivel taxonómico, 1150 de aves (57\% determinados) y 1401 de peces (71\%).
La pesca domina con $46 \%$ de los restos, pero esta actividad esta representada sobretodo en un sitio, Dawson 3, tanto en la terraza alta como en la terraza baja, y muy poco en los otros sitios (Fig. 4).

Las aves (38\%) aparecen en segunda posición y de manera más regular que los peces: están presentes en casi todos los sitios. Sin embargo, la importancia

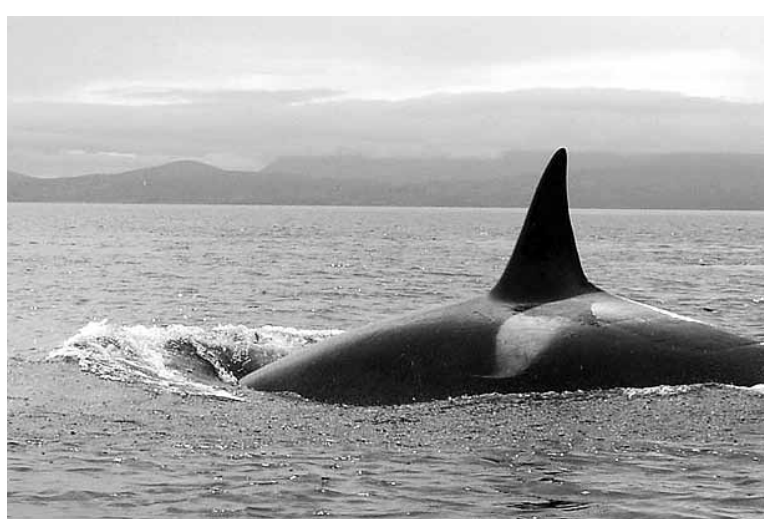

Fig. 3. Orca macho juvenil avistada en las proximidades de isla Wickham (D. Legoupil).

Tabla 1. Los restos de fauna vertebrada en los sitios estudiados (NR2).

\begin{tabular}{|c|c|c|c|c|c|c|c|}
\hline Familia & $\begin{array}{c}\text { Dawson 3 } \\
\text { A1-2 }\end{array}$ & $\begin{array}{c}\text { Dawson 3 } \\
\text { A30 }\end{array}$ & Dawson 16 & Wickham 1 & Wickham 2 & Offing 1 & Total \\
\hline mamíferos & 92 & 27 & 98 & 28 & 31 & 198 & 474 \\
\hline aves & 654 & 75 & 244 & 37 & 62 & 78 & 1150 \\
\hline peces & 1306 & 70 & 3 & 5 & 13 & 4 & 1401 \\
\hline total & 2052 & 172 & 345 & 70 & 106 & 280 & 3025 \\
\hline
\end{tabular}

2 NR (número de restos) o en inglés NSP (number of specimens). 


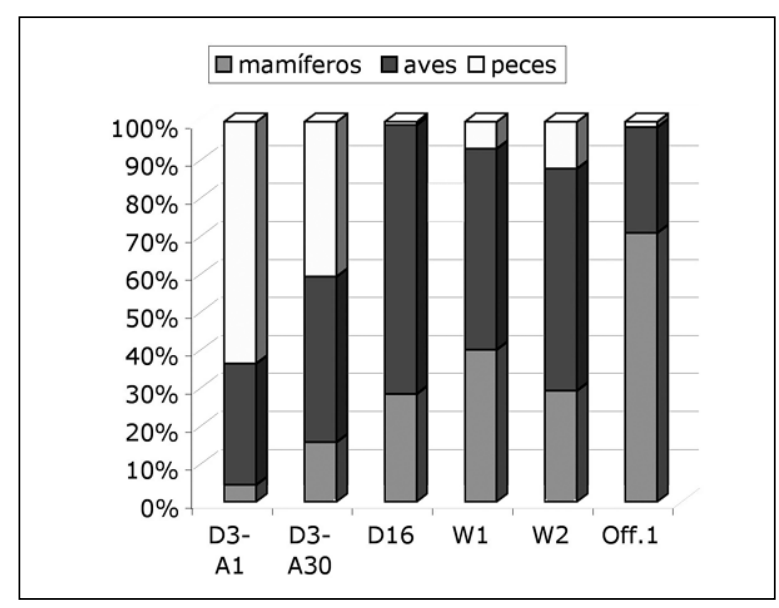

Fig. 4. Representación de los restos de fauna vertebrada (NR).

de aves y peces reducirían su papel considerablemente con relación a los grandes mamíferos, y en particular a los pinnípedos, si se considera el aporte energético y no el número de restos.

La caza de los mamíferos, muy pobre en Dawson 3, domina en un único yacimiento, Offing 1 (71\% de los restos). Por último, los restos asignados a mamíferos terrestres aparecen en muy baja frecuencia (2\%), aunque se encuentran presentes en casi todos los yacimientos detectados, con la única excepción de Offing 1.

\section{La caza de mamíferos}

Entre los mamíferos dominan los marinos (95\% de los restos), particularmente los pinnípedos (81\%) presentes en todos los yacimientos (Tabla 2).

\section{Los mamíferos marinos}

A nivel taxonómico se pudo comprobar en los diferentes sitios la presencia de las dos especies de pinnípedos, destacando que los restos de Arctocephalus australis dominan en la zona meridional (sondeo A1/2 de Dawson 3), y los de Otaria flavescens en la zona central (Dawson 16 y Offing 1).

Esta caza, omnipresente pero ocasional, constituyó una actividad especializada en Offing 1. La mayoría de los restos corresponden a individuos adultos, entre los cuales se identificó al menos a un gran macho Otaria flavescens, representado por una decena de restos. Se encontraron también en este sitio restos de juveniles sin una asignación precisa de edad y 3 restos que representaban al menos a un neonato, evidencia que permite asignarle un carácter estival a, por lo menos, una fase de ocupación del sitio.

La presencia de un apostadero de lobos en la costa noreste del islote Offing podría explicar la alta incidencia de pinnípedos en este yacimiento. Sin embargo, a poca distancia y en un contexto casi similar, el sitio Offing 2 presenta un registro faunístico más diversificado durante los cerca de tres milenios en los que fuera ocupado, atestiguando eventos de caza de albatros (Diomedea sp.) y una importante actividad de pesca (Legoupil et al. 2009 y Torres 2010).

En relación a otros mamíferos, debemos indicar que los delfines, si bien aparecen en el registro, están escasamente representados, destacando el hallazgo de un segmento de columna vertebral en conexión anatómica en el sitio Wickham 1.

Los mustélidos, cuyo hábitat se corresponde bien con las costas rocosas del sur, han sido cazados principalmente en el canal Meskem. En Dawson 3

Tabla 2. Los restos de mamíferos (NRD $\left.{ }^{3}\right)$.

\begin{tabular}{|c|c|c|c|c|c|c|c|c|}
\hline Familia & Especie & $\begin{array}{c}\text { Dawson 3 } \\
\text { A1-2 }\end{array}$ & $\begin{array}{c}\text { Dawson 3 } \\
\text { A30 }\end{array}$ & Dawson 16 & Wickham 1 & Wickham 2 & Offing 1 & Total \\
\hline Cetáceos & indet & & & 2 & & 2 & 4 \\
\hline Pinnípedos & indet. & 42 & 19 & 68 & & 11 & 167 & 307 \\
\hline & O. flavescens & 1 & 3 & 17 & 1 & 6 & 10 & 38 \\
\hline & A. australis & 12 & & 1 & 1 & & 14 \\
\hline Delfínidos & Delphin sp. & 4 & 1 & 1 & 23 & & 29 \\
\hline Mustélidos & Lontra sp. & 26 & 1 & & & 2 & 1 & 30 \\
\hline Artiodáctilos & indet. & 4 & & 4 & & 6 & 1 & 15 \\
\hline & L. guanicoe & 2 & 2 & 2 & & 2 & & 8 \\
\hline T. mamíferos & & 91 & 26 & 95 & 25 & 29 & 179 & 445 \\
\hline
\end{tabular}

3 NRD (número de restos determinados) o en inglés NISP (number of identified spicemens). 
(A1/2), se registró una treintena de restos distribuidos en diferentes capas, alcanzando hasta unos sesenta centímetros de profundidad, bastante cerca de la capa basal datada en 3000 años AP. La caza de nutrias, actividad registrada en yacimientos tardíos como Punta Baja (Legoupil 1989) o en el archipiélago de Cabo de Hornos (Legoupil 1993/94), se encuentra igualmente descrita por la etnografía (Hyades 1891, Gusinde 1986). Los hallazgos realizados en Dawson 3 permiten corroborar que la caza de nutrias fue practicada desde al menos 3000 años AP, extendiendo considerablemente la cronología conocida para dicha actividad puntual.

Nuevas excavaciones en el sitio Dawson 3 permitirían quizá definir un terminus ante quem para esta pequeña actividad cinegética cuyo objetivo era -según las fuentes etnográficas- la recuperación de las pieles y no el consumo de carne.

Finalmente, se registraron algunos fragmentos de hueso de cetáceo en los sitios Dawson 16 y Wickham 2. Estos elementos no estarían necesariamente ligados al consumo de éstos animales. Todos los restos corresponden a costillas y podrían haber sido recolectados como materia prima para fabricar herramientas.

Los guanacos: evidencia de contactos limitados pero regulares con Tierra del Fuego

No existe ninguna evidencia de la existencia de guanacos (Lama guanicoe) o huemules (Hippocamelus bisulcus) en isla Dawson. Sin embargo, las zonas de praderas del norte eran favorables a los primeros y los relieves montañosos y pantanos del sur a los segundos. La isla no habría sido accesible por vía terrestre en ningún momento ni del tardiglacial ni durante el Holoceno temprano (Clapperton 1992). Además, la distancia que la separaba, tanto del continente como de Tierra de Fuego -una decena de kilómetros- habría hecho muy difícil la travesía a nado para ambas especies. Sin embargo, se registraron huesos de guanaco en casi todos los yacimientos ${ }^{4}$, con excepción del sitio Offing 1.

Los escasos restos de guanaco probablemente fueron transportados, quizá en porciones o tal vez como elementos aislados $y / o$ instrumentos. La hipótesis tecnológica está fundada por el tipo de elementos representados en los yacimientos,

4 Corresponden a 23 restos que incluyen 5 artefactos. que corresponden exclusivamente a fragmentos de huesos largos, destacando que en 5 casos estos se encuentran manufacturados. Es probable que estos restos fueran colectados en Tierra del Fuego donde los guanacos son abundantes. La hipótesis de un origen continental es menos plausible dada la mayor distancia a la que se encuentra la península Brunswick -5 a 10 veces más- en el caso que deba contornearse la costa de Dawson antes de cruzar el estrecho de Magallanes.

Pese a su gran superficie, la presencia de hábitats adecuados para albergar poblaciones de guanacos y huemules y la proximidad a Tierra del Fuego, la isla Dawson se inscribe exclusivamente en el universo marítimo de los canoeros. Sin embargo, sus ocupantes habrían tenido contacto con la Tierra del Fuego. Este contacto se encuentra representado en los diferentes sitios arqueológicos de isla Dawson, mostrando evidencias para todas las épocas representadas.

La caza de aves: Una explotación omnipresente de cormoranes y otros eventos de caza

Aunque el aporte energético es bajo, las aves están bien representadas en el conjunto de sitios muestreados donde éstas constituyen el 81\% del total de restos de tetrápodos recuperados. Una veintena de taxa fueron identificados (Tabla 3):

- Sphenisciformes: dos especies de pingüinos (Spheniscus magellanicus y Eudyptes chrysocome),

- Procellariiformes: un albatros (probablemente de ceja negra) (Diomedea cf. melanophris) y un petrel (Procellaria sp.),

- Pelecaniformes: dos especies de cormoranes (Phalacrocorax atriceps y P. magellanicus)

- Anseriformes: cuatro especies que incluyen caiquén, caranca y canquén (Chloephaga picta, $C$. hybrida, C. poliocephala y C. rubidiceps), el pato quetro (Tachyeres sp.), el pato juarjual (Lophonetta specularioides) y un pato rana (Oxyura sp.),

- el tiuque (Milvago chimango),

- Charadriiformes: el queltehue (Vanellus chilensis), la gaviota dominicana (Larus dominicanus) y la gaviota austral (Leucophaeus scoresbii), el salteador pardo (Stercorarius antarcticus),

- Strigiformes: el concón (Strix rufipes),

- Piciformes: el carpintero negro (Campephilus magellanicus). 
Estos taxa no aparecen en todos los sitios considerados aquí: sin sorpresa observamos que los sitios que poseen la mayor cantidad de restos Dawson 3 (A1-A2) y Dawson 16- son los que poseen una mayor diversidad taxonómica.

Cormoranes, anátidos y pingüinos están presentes en todos los sitios, con proporciones totales de 52\%, 22\% y 6\% respectivamente. Sin embargo, estas proporciones varían según los sitios. En Offing 1 se observa la proporción más importante de cormoranes, que representan el $81 \%$ del total de aves determinadas. Los cormoranes son igualmente mayoritarios en el sondeo A1-2 de Dawson 3 (45\%), en Dawson 16 (66\%) y en Wickham 2 (66\%).

En los dos sitios en donde los cormoranes no son dominantes en el conjunto de avifauna, el sondeo A30 de Dawson 3 y en Wickham 1, el primer lugar es ocupado por anátidos, con un $50 \%$ y un $77 \%$ respectivamente para cada sitio.
La presencia de pingüinos es importante en Wickham 2 , en donde representan el taxón más abundante después de los cormoranes con un 24\% de restos determinados. En Wickham 1, estos son numerosos tanto como los cormoranes (11,5\%). En el sondeo A30 de Dawson 3, en Dawson 16 y en Offing 1 , estos se ubican en tercera posición, pero en los sondeos de la terraza alta de Dawson 3 (en A1-2), es uno de los taxa menos representados.

Los otros taxa representan menos del 7\% de los restos de aves determinadas. No obstante dos excepciones deben tenerse en cuenta, ambas observadas en el sondeo A1-2 de Dawson, en donde los albatros y queltehues representan, respectivamente, el $10 \%$ y $8 \%$ de los restos determinados. Una caza muy específica sobre albatros fue igualmente registrada en el sitio de Offing 2 , yacimiento que es actualmente objeto de excavaciones extensivas. (Lefèvre et al. 2010).

Tabla 3. Los restos de avifauna de los sitios estudiados.

\begin{tabular}{|c|c|c|c|c|c|c|c|c|c|c|c|c|c|c|c|c|}
\hline \multirow[t]{2}{*}{ Orden } & \multirow[t]{2}{*}{ Familia } & \multirow[t]{2}{*}{ Especie } & \multicolumn{2}{|c|}{$\begin{array}{c}\text { Dawson } 3 \\
\text { A1-2 }\end{array}$} & \multicolumn{2}{|c|}{$\begin{array}{c}\text { Dawson } 3 \\
\text { A30 }\end{array}$} & \multicolumn{2}{|c|}{ Dawson 16} & \multicolumn{2}{|c|}{ Wickham 1} & \multicolumn{2}{|c|}{ Wickham 2} & \multicolumn{2}{|c|}{ Offing 1} & \multicolumn{2}{|c|}{ Total } \\
\hline & & & NR & $\%$ & NR & $\%$ & NR & $\%$ & NR & $\%$ & NR & $\%$ & NR & $\%$ & NR & $\%$ \\
\hline Sphenisciformes & Spheniscidae & & 4 & 1,2 & 6 & 14,3 & 16 & 10,5 & 3 & 11,5 & 9 & 23,7 & 4 & 5,9 & 42 & 6,4 \\
\hline Procellariiformes & Diomedeidae & Diomedea sp. & 34 & 10,2 & 1 & 2,4 & 7 & 4,6 & & & & & & & 42 & 6,4 \\
\hline Procellariiformes & Procellariidae & Procellaria sp. & & & & & 1 & 0,7 & & & & & & & 1 & 0,2 \\
\hline Pelecaniformes & Phalacrocoracidae & $\begin{array}{l}\text { Phalacrocorax } \\
\text { sp. }\end{array}$ & 148 & 44,6 & 13 & 31,0 & 100 & 65,8 & 3 & 11,5 & 25 & 65,8 & 55 & 80,9 & 344 & 52,3 \\
\hline Anseriformes & Anatidae & & 73 & 22,0 & 21 & 50,0 & 19 & 12,5 & 20 & 76,9 & 3 & 7,9 & 9 & 13,2 & 145 & 22,0 \\
\hline Falconiformes & Falconidae & $\begin{array}{l}\text { Milvago } \\
\text { chimango }\end{array}$ & & & & & & & & & 1 & 2,6 & & & 1 & 0,2 \\
\hline \multirow[t]{5}{*}{ Charadriiformes } & Charadriidae & $\begin{array}{l}\text { Vanellus } \\
\text { chilensis }\end{array}$ & 27 & 8,1 & & & & & & & & & & & 27 & 4,1 \\
\hline & $\begin{array}{l}\text { Charadriidae / } \\
\text { Scolopacidae }\end{array}$ & & 18 & 5,4 & & & & & & & & & & & 18 & 2,7 \\
\hline & Laridae & $\begin{array}{l}\text { Larus } \\
\text { dominicanus }\end{array}$ & 22 & 6,6 & & & 1 & 0,7 & & & & & & & 23 & 3,5 \\
\hline & Laridae & $\begin{array}{l}\text { Leucophaeus } \\
\text { scoresbii }\end{array}$ & 3 & 0,9 & & & 2 & 1,3 & & & & & & & 5 & 0,8 \\
\hline & Stercorariidae & $\begin{array}{l}\text { Stercorarius } \\
\text { skua }\end{array}$ & 3 & 0,9 & 1 & 2,4 & & & & & & & & & 4 & 0,6 \\
\hline Strigiformes & Strigidae & Strix rufipes & & & & & 4 & 2,6 & & & & & & & 4 & 0,6 \\
\hline Piciformes & Picidae & $\begin{array}{l}\text { Campephilus } \\
\text { magellanicus }\end{array}$ & & & & & 2 & 1,3 & & & & & & & 2 & 0,3 \\
\hline $\begin{array}{l}\text { Total } \\
\text { identificados }\end{array}$ & & & 332 & 50,8 & 42 & 56,0 & 152 & 62,3 & 26 & 70,3 & 38 & 61,3 & 68 & 87,2 & 658 & 57,2 \\
\hline Indeterminados & & & 252 & 38,5 & 31 & 41,3 & 66 & 27,0 & 10 & 27,0 & 19 & 30,6 & 6 & 7,7 & 384 & 33,4 \\
\hline $\begin{array}{l}\text { Juveniles } \\
\text { indeterminados }\end{array}$ & & & 70 & 10,7 & 2 & 2,7 & 26 & 10,7 & 1 & 2,7 & 5 & 8,1 & 4 & 5,1 & 108 & 9,4 \\
\hline Total Aves & & & 654 & & 75 & & 244 & & 37 & & 62 & & 78 & & 1150 & \\
\hline
\end{tabular}


Es interesante destacar que en casi todos los sitios incluidos en el muestreo se registraron restos de aves juveniles. Estas representan el 27\% del total de restos indeterminados para el conjunto de sitios, pero esta proporción varía según el yacimiento. La proporción más importante de juveniles se registra en Offing 1 donde se eleva al 67\%. En los dos sondeos de Dawson 3, alcanza un 28\% para el sondeo de la terraza alta y sólo un 6\% para el sondeo efectuado en la terraza baja. En los sitios Wickham 1 y 2, las frecuencias son del 10\% y 26\% respectivamente. En el sitio Dawson 16, la frecuencia de juveniles llega al 35\%.

En síntesis, se observan situaciones muy variables de un sitio al otro, a veces con cambios en los taxa dominantes, sin que sea posible vincular estas preferencias a un medio-ambiente que sería más favorable a una especie por sobre otra. Es igualmente difícil de interpretar las diferencias observadas en función de las antigüedades de los sitios.

Si se compara la caza de aves entre la isla Dawson y el canal Beagle, los análisis conducidos por Tivoli (2010a, b, c) indican una composición bastante similar del espectro avifaunístico: une fuerte presencia de los cormoranes, especialmente en los componentes antiguos de los sitios, con una excepción en el nivel $\mathrm{F}$ de Mischiuen, donde los pingüinos representan casi el $70 \%$ de los restos. En segundo lugar vienen los anátidos, los albatros/ Procellariidae grandes, o los pingüinos. En los componentes recientes, Tivoli observa un cambio, con un aumento de la frecuencia de restos de aves en relación a otros animales, y un porcentaje más importante de los Diomedeidae y Procellariidae, con la excepción de Túnel II, donde los cormoranes representan casi $90 \%$ del conjunto.

\section{Los moluscos: un rol limitado salvo en la zona sur y sobre el islote Offing}

Los restos de conchas están presentes en casi todos los sitios, pero de manera muy desigual. Sus acumulaciones son muy limitadas en sitios de la costa este de isla Dawson, no permitiendo la formación de depósitos espesos, salvo en Offing 2.

Por el contrario, hacia la porción sur, en el canal Meskem, se observaron importantes acumulaciones de conchas. En particular, en el sitio Dawson 3 , en donde se registran diferencias significativas

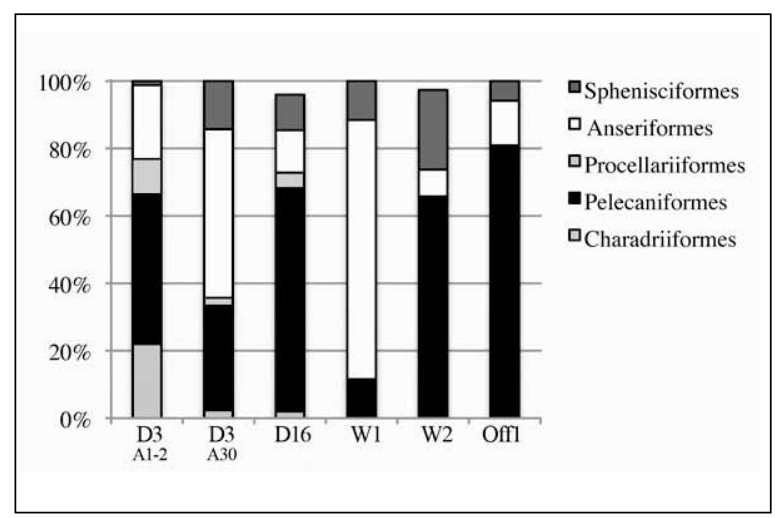

Fig. 5. Representación de los distintos grupos de aves.

entre los conchales emplazados en las diferentes terrazas que ocupa el yacimiento. Sobre la terraza de 4 msnm, datada en circa 3000 años AP, en un total de aproximadamente 5000 restos determinados en una columna de $1,5 \mathrm{~m}^{2}$, los moluscos más representados corresponden a Mytilus edulis chilensis (78,6\%), Venus antiqua (10,8\%), Nacella sp. $(4,3 \%)$ y Acanthina monodon (2,4\%) (K. Salas en Legoupil et al. 2007). Las especies del mesolitoral rocoso (mitílidos y gasterópodos) dominan largamente el conjunto, pero están igualmente asociadas a una pequeña actividad de colecta de bivalvos de sustrato arenoso. En el depósito de conchal de la terraza baja, sobre una muestra de 1170 restos, sólo aparecen representadas especies características del litoral rocoso, particularmente Mytilus edulis (48,5\%), Nacella sp. (18,7\%) y Trophon sp. (13,2\%) (ibid.). Si la ausencia de bivalvos característicos de fondos arenosos, como por ejemplo las almejas, se confirma para el depósito de esta terraza, se podría interpretar como evidencia de un cambio del comportamiento de los ocupantes del sitio en época tardía, o bien, un cambio microambiental local afectando la disponibilidad de dichos taxa.

El sitio Wickham 2, emplazado inmediatamente al frente de Dawson 3, corresponde a un espeso conchal constituido por el ensamble de especies típicas (mitílidos y gasterópodos), destacando que se registraron también numerosas valvas de ostión del sur (Pectinidae). Estos bivalvos, raramente descritos en los conchales de cazadores marinos, atestiguarían una colecta a través del buceo, práctica descrita entre los canoeros por navegantes durante el siglo XVII (Duplessis 2003). 


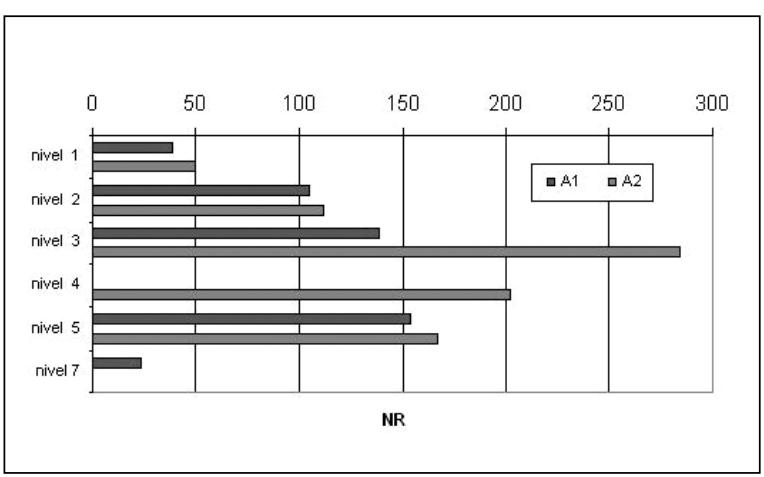

Fig. 6. Distribución de los restos de peces en la estratigrafía de las unidades A1 y A2 del sitio Dawson 3.

\section{Una pesca orientada hacia la captura de peces de profundidad}

Un total de 1401 restos de peces fueron recolectados en los sitios de la isla Dawson (P. Béarez en Legoupil et al. 2007), pero la casi totalidad de ellos se concentra en el sitio Dawson 3, por lo cual es el único sitio aquí considerado. La muestra estudiada proviene de un sondeo de $2 \mathrm{~m}^{2}$ (A1 y A2) ubicado sobre la terraza de $4 \mathrm{msnm}$ (Legoupil et al. 2011). Si bien el conchal muestra un espesor de cerca 1 $\mathrm{m}$, los restos de peces se distribuyen hasta $70 \mathrm{~cm}$ de profundidad, principalmente en los 5 primeros niveles (Fig. 6).

Los dos $\mathrm{m}^{2}$ del sondeo se analizaron como una sola unidad. Se identificaron los restos al mejor nivel taxonómico posible y se cuantificó el número mínimo de individuos (NMI). Un total de 1306 restos de peces fue revisado, del cual un porcentaje muy significativo (72,7\%) fue identificado taxonómicamente.

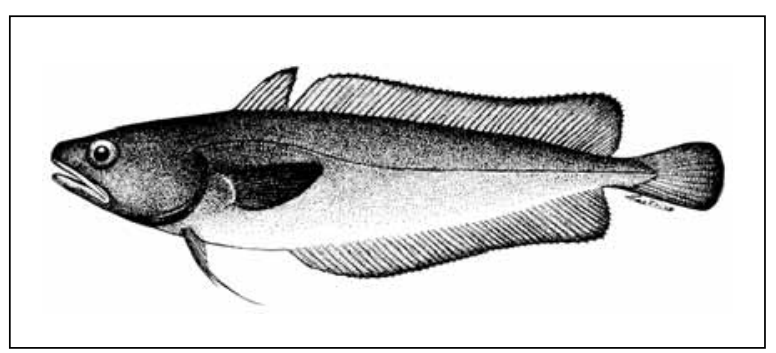

Fig. 7. La brótula, Salilota australis (Fuente: FAO)

Diversidad taxonómica

La diversidad encontrada es bastante alta para la zona con ocho taxa reconocidos (Tabla 4). La especie mayormente representada es la brótula (Salilota australis: 77,6\%) y en segundo lugar el congrio dorado (Genypterus blacodes : 16,9\%). Ambas son especies demersales, es decir ligadas al fondo. La primera (Fig. 7) es una especie que habita en ambientes de fondo blando o duro entre 10 y 900 m de profundidad (Cassia y Hansen 2005; Lloris y Rucabado 1991). En los canales magallánicos se señala su presencia en el ambiente sublitoral rocoso entre 25 y 35 m de profundidad (Sielfeld et al. 2006). La segunda es una especie de similar hábitat, viviendo entre 10 y 400 m (Lloris y Rucabado 1991). La cabrilla (Sebastes oculatus) vive sobre el fondo en zonas rocosas a partir de los 10 $m$ de profundidad (Pequeño y Riedemann 2006) y hasta más de 100 m (Lloris y Rucabado 1991). En cambio, la merluza de tres aletas (Micromesistius australis) tiene un comportamiento bento-pelágico y se sabe muy poco sobre su presencia cerca del litoral. Cabe señalar aquí que esta última especie se registra por primera vez en un sitio arqueológico de la Patagonia.

Tabla 4. Diversidad taxonómica y abundancia de los peces en Dawson 3 unidades A1/A2.

\begin{tabular}{|c|c|c|c|c|c|c|}
\hline Taxa & NR & $\%$ & NMI & $\%$ & Peso & $\%$ \\
\hline Salilota australis (brótula) & 736 & 77,56 & 42 & 70 & 287,27 & 56,6 \\
\hline Genypterus blacodes (congrio dorado) & 160 & 16,86 & 5 & 8,33 & 204,07 & 40,21 \\
\hline Eleginops maclovinus (róbalo) & 23 & 2,42 & 3 & 5 & 7,16 & 1,41 \\
\hline Cottoperca gobio (toro de los canales) & 13 & 1,37 & 3 & 5 & 3,04 & 0,6 \\
\hline Sebastes oculatus (cabrilla) & 9 & 0,95 & 3 & 5 & 5,05 & 0,99 \\
\hline Micromesistius australis (merluza de tres aletas) & 3 & 0,32 & 2 & 3,33 & 0,71 & 0,14 \\
\hline Chondrichthyes (peces cartilaginosos) & 4 & 0,42 & 1 & 1,67 & 0,22 & 0,04 \\
\hline Nototheniidae (tramas) & 1 & 0,1 & 1 & 1,67 & 0,05 & 0,01 \\
\hline Total determinados & 949 & 100 & 60 & 100 & 507,57 & 100 \\
\hline Indeterminados & 357 & - & - & - & 91,56 & - \\
\hline Total & 1306 & & 60 & & 584,74 & \\
\hline
\end{tabular}


Todas estas especies pueden encontrarse en profundidades similares y entonces pudieron ser capturadas en el mismo lugar y ambiente de pesca, aunque no necesariamente a mucha distancia de la orilla, especialmente en el periodo estival cuando se aproximan a la costa.

Los otros taxa presentes, aunque escasos, representan otros ambientes explorados: el intermareal o submareal rocoso y las desembocaduras de ríos. El toro de los canales (Cottoperca gobio) es una especie bentónica litoral que pudo ser capturada en aguas someras (López et al. 1996, Sielfield et al. 2006, Pequeño y Riedemann 2005), aunque Lloris y Rucabado (1991) indican su presencia hasta $270 \mathrm{~m}$ de profundidad. Por otro lado, la presencia de róbalo (Eleginops maclovinus) muestra con claridad eventos de pesca de orilla, ya que las aguas mixohalinas favorecen la concentración de esta especie en aguas someras de desembocaduras de ríos (Guzmán y Campodónico 1973). Próximo a Punta Cannon hay un riachuelo pequeño que permitiría estas condiciones, además en esta zona de fiordo hay abundantes desagües de agua dulce.

\section{Distribución de peso}

Se reconstituyeron los pesos de los individuos enteros sobre la base de la comparación de los tamaños de los restos con los de los especimenes de la colección osteológica de referencia. El peso de los ejemplares de Salilota australis promedia $1,9 \mathrm{~kg}$, pero se observan individuos desde muy pequeños $(0,4 \mathrm{~kg})$ hasta $4 \mathrm{~kg}$. Actualmente en la zona de Punta Arenas, según nuestras observaciones en las proximidades de Punta Carrera, el peso de las brótulas difícilmente sobrepasa los $2 \mathrm{~kg}$. No obstante, estudios actuales de la población de $S$. australis en el océano atlántico suroeste (Cassia y Hansen 2005), indican que las tallas más frecuentes están comprendidas entre 38 y $54 \mathrm{~cm}$ de longitud total $(0,5-1,5 \mathrm{~kg})$, pero que la especie alcanza una talla máxima de 80 $\mathrm{cm}(4 \mathrm{~kg})$. En el caso de Genypterus blacodes, se observan individuos todavía más grandes, con un peso promedio de aproximadamente $5 \mathrm{~kg}$ y pesos máximos evaluados entre 10 y $12 \mathrm{~kg}$. En la actualidad, según Nielsen et al. (1999), el congrio dorado puede alcanzar un tamaño de $2 \mathrm{~m}$ por unos $25 \mathrm{~kg}$.

\section{Representación de unidades anatómicas}

La brótula esta más representaba por restos craneales (401) que del postcráneo (331 vértebras).
Los elementos más abundantes son el basioccipital y el parasfenoides, probablemente se preservaron juntos al tener directa conexión anatómica, pues forman la base ventral y posterior del cráneo. Igualmente, dentarios, articulares y maxilares tienen buena representación. En el caso del congrio dorado, se observa una mayor presencia de vértebras, las cuales representan los 2/3 de los restos de la especie.

La ictiofauna representada en el sitio Dawson 3 indica una pesca dirigida a especies de gran tamaño que se mueven en ambientes de fondo y a una profundidad de varios metros como el congrio dorado, la brótula y en menor medida la merluza de tres aletas.

En cambio los recursos de orilla muestran baja importancia. Hay escasa explotación de pequeños peces, tal como los nototénidos, que habitan microambientes de intermareal y submareal rocoso o de playas de bloques y cantos, característicos de la costa occidental del estrecho de Magallanes y del canal Whiteside. Igualmente, los pocos restos de róbalo muestran una poca orientación hacia la pesca en ambientes estuarinos.

Las implicaciones de la presencia de las tres primeras especies son importantes en relación al nivel tecnológico pesquero de los ocupantes del sitio en esta época. Brótulas y congrios, cuando adultos, tienen un régimen alimenticio ictiófago, y es probable que fueran capturados con líneas de mano y cebo de carne o pescado. Aunque estas especies se acercan a la orilla en verano (Lloris y Rucabado 1991; López et al. 1996), nos inclinamos a pensar que la estrategia de pesca se orientó a ambientes de profundidad, no necesariamente a mucha distancia de la costa, si tomamos en cuenta que las tallas de los individuos son especialmente grandes y superan los tamaños usualmente capturados en la orilla. Esta preferencia en la explotación de peces de ambientes de mayor profundidad y tamaño, también se respalda en que pese a que el ambiente rocoso de orilla es explotado intensivamente en este sitio para la recolección de moluscos, ya que la mayoría corresponde a estos ambientes, los peces de este ambiente se encuentran escasamente representados.

En cuanto a las evidencias de tecnología de pesca de los sitios del periodo comprendido entre los 3500 y 2500 años AP, no hay claridad del uso de un tipo de técnica. No obstante se han encontrado pequeñas puntas óseas que podrían ser parte de instrumentos para la pesca (Legoupil et al. 2011). 


\section{CONCLUSIÓN}

Una primera constatación sobre la economía de subsistencia de los ocupantes de isla Dawson e islas aledañas es que esta estuvo basada prioritariamente en recursos marinos. Los restos de mamíferos terrestres, específicamente los guanacos, si bien aparecen en los sitios de diferente cronología, corresponden a evidencias muy escasas. Probablemente sus restos pueden ser explicados como producto de la visita o estadía de estos grupos en las costas de la isla Tierra del Fuego. De todas maneras, la evidencia permite sostener que isla Dawson no fue poblada por camélidos ni cérvidos en ningún momento del Holoceno. En relación a esto, debemos destacar que la explotación de mamíferos terrestres es, hasta el momento, una actividad pobremente representada entre cazadores marinos del estrecho de Magallanes, en particular si uno observa los registros obtenidos para los sitios Tempranos de Bahía Buena 1 y Punta Santa Ana 1, ubicados en península Brunswick, en donde las evidencias refrendan una explotación muy restringida y circunstancial para las dichas especies de disponibilidad de (cf. San Román 2007, 2010).

En relación a la caza de mamíferos marinos, destacamos el predominio de los lobos marinos, presentes en todos los sitios, como recurso principal. Es probable que las tendencias observadas en cuanto a la distribución de especies y emplazamiento geográfico de los sitios tenga relación con la disponibilidad natural de las dos especies registradas: Otaria flavescens en la porción central de Dawson y Arctocephalus australis en el sur, aunque el tamaño de las excavaciones no permite aun confirmar de manera consistente esta observación.

Frente a un abanico taxonómico muy diversificado en la mayoría de los sitios, dos se destacan por su fuerte especialización: Offing 1 en la caza de los lobos y Dawson 3 en la pesca asociada con la caza de aves.

La caza de los lobos en Offing 1 podría justificarse por la presencia de un apostadero de lobos en la porción nororiental del islote donde uno esta visible actualmente. Esto plantea un interesante tema de investigación referido a la relación entre sitios de campamentos y lugares de caza, y también al estudio de la etología de estos animales y a sus variaciones en el tiempo. Este sitio presenta una segunda particularidad: la fuerte asociación entre caza de lobos y explotación de cormoranes, ambas taxa representando el 95\% del total de los restos. Un modelo idéntico ya fue observado en el mar de Otway: en Bahía Colorada para el momento temprano (Legoupil 1997), en Punta Baja para el periodo tardío (Legoupil 1989). Con el tercer ejemplo del sitio intermedio de Offing 1 ( $\pm 3000 \mathrm{BP})$, este modelo merecería ser profundizado en función de la estacionalidad de ocupación de los sitios, de las especies presentes y de los nichos ecológicos representados.

El segundo sitio especializado, Dawson 3, se destaca por la explotación de aves (en particular cormoranes, anátidos, albatros, gaviotas, entre otros) y la pesca (brótulas y congrios).

La pesca de grandes peces de fondo, posiblemente con líneas de mano es una característica que permite vincular tres sitios con fechados de principios del primer milenio AC: Dawson 3, Offing 2 y Punta Santa Ana 2 (Legoupil et al. 2007, 2009; San Román et al. 2009). Estos tres sitios se caracterizan por la pesca orientada hacia peces de gran tamaño que se desplazan a mayor profundidad, tales como el congrio, la brótula y, en menor medida, la merluza de tres aletas (Torres, 2010). No obstante hay que aclarar que este es un patrón de explotación haliéutico que se viene desarrollando desde las primeras ocupaciones de grupos canoeros de esta zona, donde también hay un predominio de la pesca de brótula, aunque quizás las técnicas de pesca pudieron haber variado, en vista de la asociación y abundancia de pesas líticas usadas posiblemente para la pesca en estos contextos tempranos (Torres y Ruz 2011).

Esta pesca específica es notablemente diferente de la practicada hace unos 3000 años atrás en otras partes de los archipiélagos, y especialmente en el canal Beagle donde, según Zangrando (2009), abundan predominantemente las merluzas (Merluccius sp.) y la merluza de cola (Macruronus magellanicus).

En el plano cronológico, se debe notar la asociación de albatros y gaviotas en la terraza superior de Dawson 3 (circa 3000 años AP), especies que han sido registradas recurrentemente en otros sitios de una antigüedad semejante, tales como Punta Santa Ana 2 (San Román et al. 2009) y Offing 2 Locus 1 (Legoupil et al. 2007; Legoupil (dir.) en prep). Entonces la caza de estas grandes aves y en menor medida la pesca de brótula podría ser la característica que mejor representa la zona del centro del estrecho de Magallanes hace tres mil años. 
Estos datos nos plantean la necesidad de evaluar si las tendencias observadas pueden ser explicadas como producto de la disponibilidad natural de aves y peces en las distintas localidades (disponibilidad estacional), o bien, como resultado de la selección o preferencia cultural por una u otra especie para las diferentes épocas representadas por los sitios estudiados.

Los recursos malacológicos explotados en Dawson e islas adyacentes muestran un abanico taxonómico típico para los depósitos de conchal registrados en otras zonas del archipiélago patagónico y fueguino. Los conjuntos analizados muestran una explotación orientada a recursos del intermareal, presentando variaciones inter-sitios que reflejarían cambios en la disponibilidad y/o frecuencia local de especies. El único caso en donde se ha observado un cambio en la explotación de moluscos corresponde al sitio Dawson 3, desaparecen cuando los bivalvos de sustrato arenoso del componente tardío del yacimiento. Evaluar si esto se debe a causas naturales - cambio en la disponibilidad de estos taxa a escala local- o a elecciones culturales, requerirá profundizar el estudio del sitio y su entorno.

Un dato de interés, relacionado con la explotación de moluscos, tiene relación con el registro de ostiones (Pectinidae) en el sitio Wickham 2. Este taxón es muy poco frecuente en sitios de cazadores marinos y podría implicar la práctica del buceo. Además, aunque por confirmar, no sería éste un recurso local $^{5}$, por lo que puede ser evidencia de explotación de bancos de moluscos con un transporte a distancia de dichos recursos.

Finalmente, las estrategias de aprovisionamiento observadas testimonian, como en otros sitios de los archipiélagos, una organización fundada sobre una mayoría de sitios con economía diversificada y algunos sitios más especializados: Dawson 3, con una asociación de caza de aves (en parte en verano) y pesca, y Offing 1 con la caza de pinnípedos y secundariamente de cormoranes. Queda por comprobar esta alternancia y determinar el ritmo de su ciclo estacional.

\section{AGRADECIMIENTOS}

Al ministerio de asuntos extranjeros francés (Departamento de Ciencias Humanas, Sociales y

5 Los bancos de ostiones actualmente explotados en la zona se ubican hacia el sur, en las inmediaciones de fiordo Parry, a aproximadamente $70 \mathrm{~km}$ de distancia de Wickham 2 .
Arqueología) por el financiamiento de los trabajos de terreno. Al proyecto FONDECYT 1085329 y el Museo de Historia Natural (Paris) por su contribución durante el estudio del material y la elaboración de esta publicación.

\section{BIBLIOGRAFÍA}

CASSIA M.C. y J. HANSEN. 2005. Distribución estacional $y$ estructura poblacional del bacalao austral (Pisces, Moridae, Salilota australis) en la zona económica exclusiva Argentina entre los $45^{\circ} \mathrm{S}$ y $56^{\circ} \mathrm{S}$ durante 1992 y 1993. Revista de Investigación y Desarrollo Pesquero 17: 55-65.

CLAPPERTON C. M. 1992. La última glaciación y desglaciación en el Estrecho de Magallanes: implicaciones para el poblamiento de Tierra del Fuego. Anales del Instituto de la Patagonia, Ser. Cs. Hs, Punta Arenas 21:113-128.

DUPLESSIS. 2003. Périple de Beauchesne à la Terre de Feu (1698-1701): une expédition mandatée par Louis XIV, texte établi et annoté par J. Bloch et présenté par M. Foucard. Transboréal, Paris

GUSINDE M. 1986. Los Indios des Tierra del Fuego. T. 2: Los Yamana. Centro Argentino de Etnología Americana ed., Buenos Aires.

GUZMÁN, L. y G. I. CAMPODÓNICO. 1973. Algunos aspectos de la biología de Eleginops maclovinus (Cuv. y Val.) 1830, con especial referencia a su morfometría, caracteres merísticos y alimentación. Anales del Instituto de la Patagonia 4: 343-371.

HYADES, 1891. Anthropologie-Ethnographie. Mission Scientifique du Cap-Horn 1882-1883. T. VII, GauthiersVillars, Paris.

LEFÈVRE C., V. LAROULANDIE y M. CHRISTENSEN 2010. Birdskin clothing in southern Patagonia. Comunicación presentada al 11 Congreso internacional del ICAZ, 2328 de Agosto, Paris.

LEGOUPIL D. (dir.). 1989. Ethno-archéologie dans les Archipels de Patagonie : les Nomades Marins de Punta Baja. Mémoire de l'ADPF, ed. Recherches sur les Civilisations, Paris.

1997. Bahia Colorada (île d'Englefield) : les premiers chasseurs de mammifères marins de Patagonie australe. Mémoire de l'ADPF, ed. Recherches sur les Civilisations, $248 p$.

en prep. El sitio Offing 2. Locus 1, ms.

LEGOUPIL D. 1993/94. Prospección en el archipiélago del Cabo de Hornos y la costa sur de la isla Navarino: po- 
blamiento y modelos económicos. Anales del Instituto de la Patagonia. 22: 101-121.

2000. El sistema socioeconómico de los nómades del mar de Skyring (archipiélago de Patagonia). Anales del Instituto de la Patagonia, Ser. Cs. Hs, 28:81-119.

LEGOUPIL D., P. BEAREZ, M. CHRISTENSEN, M. LANGLAIS C. LEFEVRE, F. MORELLO, M. SAN ROMAN y K. SALAS. 2007. La Culture à grandes pointes : prospection archéologique sur la côte est de l'île Dawson. Rapport (Ministère des Affaires Etrangères-France), 53p. MS.

LEGOUPIL D., P. BEAREZ, P. BERTRAN, M. CHRISTENSEN, C. LEFEVRE. 2009. La Culture à grandes pointes : La fouille archéologique du site d'Offing 2 : deuxième campagne. Rapport (Ministère des Affaires EtrangèresFrance), 51p. MS.

LEGOUPIL D, M. CHRISTENSEN y F. MORELLO. 2011. Una encrucijada de caminos: el poblamiento de la isla Dawson (Estrecho de Magallanes). Magallania 39(2) :137-152.

LOPEZ, H. L., M. L. GARCIA y N. SAN ROMAN. 1996. Lista Comentada de la Ictiofauna del Canal de Beagle, Tierra del Fuego, Argentina. CADIC, contribución científica publicación especial 85. pp. Ushuaia.

LLORIS, D y J. RUCABADO. 1991. Ictiofauna del Canal de Beagle (Tierra del Fuego). Aspectos ecológicos y análisis biogeográfico. Publicaciones Especiales del Instituto Español de Oceanografía, Madrid.

NIELSEN J. G., COHEN D. M., MARKLE D. F. Y ROBINS C. R., 1999. Ophidiiform fishes of the world (Order Ophidiiformes). An annotated and illustrated catalogue of pearlfishes, cusk-eels, brotulas and other ophidiiform fishes known to date. FAO Fisheries Synopsis 125(18). Rome, FAO.

PEQUEÑO, G. y A. RIEDEMANN. 2005. Los peces bentónicos de los canales orientales de Aysén, Patagonia (XI Región, Chile), recolectados durante el crucero CIMAR 7 Fiordos. Ciencia y Tecnología del Mar, 28(1): 113-118.

PEQUEÑO, G. y A. RIEDEMANN. 2006. Peces bentónicos capturados en el crucero CIMAR 8 Fiordos, en los canales de Aysén (XI Región, Chile). Ciencia y Tecnología del Mar, 29(1): 163-172.

SAN ROMAN, M. 2007. La explotación de mamíferos en el sitio de Bahía Buena: economía de canoeros tempranos de Patagonia (estrecho de Magallanes, Chile). En Arqueología de Fuego-Patagonia. Levantando piedras, desenterrando huesos... y develando arcanos... Editado por F. Morello, A. Prieto, M. Martinic y G. Bahamonde, pp. 295-310. Ediciones CEQUA, Punta Arenas.

2010 La explotación de recursos faunísticos en el sitio Punta Santa Ana 1: Estrategias de subsistencia de grupos cazadores marinos tempranos de Patagonia meridional. Magallania 38 (1):183-198.

SAN ROMÁN, M. K. SALAS Y M. FONTUGNE 2009 Primeros avances en la reconstrucción de secuencias de ocupación de cazadores recolectores marinos en el estrecho de Magallanes, Patagonia meridional. En Arqueología de la Patagonia. Mirada desde el Último confín. Editorial Utopías, Ushuaia, pp-35-46.

SIELFELD W., G. GUZMÁN y N. AMADO. 2006. Distribución de peces del litoral rocoso de los canales patagónicos occidentales (48 $\left.37^{\prime} .53^{\circ} 34^{\prime}\right)$. Anales del Instituto de la Patagonia 34: 21-32 21.

TIVOLI, A. M. 2010a. Las aves en la organización socioeconómica de cazadores-recolectores-pescadores del extremo sur sudamericano. Tesis de Doctorado, Universidad de Buenos Aires. MS.

TIVOLI, A. M. 2010b. Temporal trend in avifaunal resource management by prehistoric sea nomads of the Beagle Channel region (southern South America). In Birds in Archaeology. Proceedings of the 6th Meeting of the ICAZ Bird Working Group in Groningen, Groningen Archaeological Studies, 10: 131-140.

TIVOLI, A. M. 2010c. Exploitation of bird resources among prehistoric sea-nomad societies of the Beagle Channel region, southern South America. Before Farming 2: $1-12$.

TORRES J. 2010. Les stratégies de pêche chez les indiens canoeros de l'extrémité australe de l'Amérique, pendant la période 2500-3500 BP: une évaluation depuis l'île Offing, l'île Dawson et Punta Santa Ana; Détroit de Magellan (Patagonie Chilienne). Mémoire de Master Préhistoire. Ethnologie. Anthropologie, de l'Univ. de Paris I Panthéon-Sorbonne. MS.

TORRES J. Y J. RUZ, 2011. Datos preliminares de la modalidad de pesca en la tradición cultural Englefield (ca. 6000 años ap), estrecho de Magallanes y mar de Otway, Región de Magallanes, Chile. Magallania 39(2) :167-178.

ZANGRANDO A. F., 2009. Historia Evolutiva y Subsistencia de Cazadores, Recolectores y Pescadores Marítimos de Tierra del Fuego: una aproximación zooarqueológica. Sociedad Argentina de Antropología, Buenos Aires. 